\title{
Inovações na Solução do Problema de Seqüenciamento em Processadores Paralelos Uniformes
}

F.M. MÜLLER, H.G. SANTOS, V.C. KÖHLER, A. MARIN ${ }^{1}$, Universidade Federal de Santa Maria, Centro de Tecnologia, Programa de Pós-Graduação em Engenharia de Produção e Curso de Ciência da Computação, 97105-900 Santa Maria, RS, Brasil.

\begin{abstract}
Resumo.
O problema abordado neste artigo trata da alocação de um conjunto finito de tarefas em um determinado número de máquinas que possuem diferentes velocidades de processamento, de forma não preemptiva, com o objetivo de minimizar o tempo total de execução das tarefas (makespan). A abordagem de solução escolhida nesse trabalho foi o método heurístico, conhecido na literatura como Kproc [7], no qual foram propostas modificações que resultaram em um ganho na qualidade de solução em 99,98\% dos tipos de instâncias considerados nos experimentos, sem um comprometimento significativo do tempo computacional. O método modificado foi denominado Iproc. Os dois métodos heurísticos mencionados foram comparados com um limitante inferior e foram implementados através do framework para problemas de otimização combinatória CORE (Combinatorial Optimization Resource Management Environment), o qual possibilita a execução remota e distribuída através da estrutura da Internet de métodos de resolução para diversos problemas de otimização combinatória. O ambiente CORE pode ser acessado em http://glover.ce.ufsm.br.
\end{abstract}

\section{Introdução}

Considerando o seqüenciamento de $n$ tarefas independentes, de forma não preemptiva, com tempos de processamento $p_{j}, j \in J=\{1, \ldots, n\}$ a $m$ máquinas paralelas uniformes $M_{1}, M_{2}, \ldots, M_{m}$ com velocidades de processamento $\sigma_{1}=1 \leq \sigma_{2} \leq \sigma_{3} \leq$ $\ldots \leq \sigma_{m}$, e sendo $T_{i}$ a soma dos tempos de processamento das tarefas alocadas no processador $M_{i}$ e $C_{i}$ o tempo total de execução do processador $M_{i}$, obtido através de $T_{i} / \sigma_{i}$, o objetivo do problema é minimizar o tempo necessário para processar todas as tarefas (makespan) definido como $C_{\max }=\max \left\{C_{i}\right\}$, para $i=1, \ldots, m$. Este problema é denominado, segundo a classificação de três campos proposta por $[6], Q \| C_{\max }$.

\footnotetext{
${ }^{1}$ felipe,haroldo,viviane,marin@inf.ufsm.br,
} 
O problema tem várias aplicações práticas tanto no ambiente industrial quanto no científico. Um exemplo pode ser encontrado num parque de máquinas onde a atualização ocorre sem a dispensa do maquinário legado. Outro exemplo pode ser o escalonamento de processos em clusters com recursos computacionais heterogêneos.

Considerando o caso em que $\sigma_{1}=\sigma_{2}=\ldots=\sigma_{m}=1$, tem-se o problema de seqüenciamento de máquinas paralelas idênticas ou $P \| C_{\max }$.

Grande parte dos métodos heurísticos utilizados para a resolução do problema $Q \| C_{\max }$ são adaptações de heurísticas inicialmente desenvolvidas para o problema $P \| C_{\max }$. As heurísticas utilizadas no presente artigo podem, de forma genérica, ser classificadas em heurísticas construtivas e heurísticas de melhoramento. Enquanto as primeiras montam, passo a passo, uma solução, as heurísticas de melhoramento tentam, através de movimentos sistemáticos, melhorar a qualidade de solução recebida.

Entre as heurísticas construtivas mais conhecidas para o $P \| C_{\text {max }}$, podemos citar a desenvolvida por [4], denominada LPT (Longest Processing Time first). A partir dessa, [3] e [1], em trabalhos independentes, propuseram uma adaptação do $L P T$ para o $Q \| C_{\max }$, outra variante do $L P T$ foi proposta por [8].

Seguindo essa idéia, [7] desenvolveram a heurística Kproc, a qual consiste em uma adaptação do algoritmo 3-Fases proposto por [9] para resolução do $P \| C_{\text {max }}$.

\section{A heurística Kproc}

A heurística Kproc baseia-se no conceito de intervalos, ou seja, as tarefas a serem alocadas são separadas de acordo com seu tamanho e o número de intervalos é definido utilizando uma função discreta que recebe como parâmetro a razão entre a quantidade de tarefas e o número de processadores disponíveis. A separação das tarefas em intervalos substitui a ordenação inicial, procedimento existente na maioria dos algoritmos tradicionais.

A estrutura intervalar utilizada pelo Kproc assegura uma alocação equilibrada de carga em cada intervalo de cada processador. Além disso, restringe o espectro de possíveis trocas vantajosas das tarefas entre os processadores.

A execução do método Kproc ocorre em três fases distintas: alocação inicial, balanceamento de carga e fase de dupla troca. Na primeira, considerada a fase construtiva do algoritmo, é efetuada a distribuição das tarefas aos processadores. Na segunda fase, parte-se da solução encontrada pela alocação inicial, e procurase a redução do tempo de finalização do processador mais carregado, transferindo tarefas deste para o processador menos carregado. Na última fase, permite-se a permutação de tarefas a partir de um alvo definido a priori, entre o processador mais carregado e os outros processadores.

Tabela 1: Função discreta para a definição do número de intervalos

\begin{tabular}{|c|c|c|c|c|c|c|c|}
\hline$n / m$ & $<10$ & {$[10,50)$} & {$[50,100)$} & {$[100,200)$} & {$[200,300)$} & {$[300,400)$} & {$[400,500)$} \\
\hline$k$ & 3 & 7 & 12 & 16 & 20 & 24 & 28 \\
\hline
\end{tabular}




\section{Fases do Kproc}

Fase 1

Passo 1 - Calcular o limitante inferior $\underset{-}{p}=\min _{j}\left\{p_{j}\right\}$ e o limitante superior $\bar{p}=$ $\max _{j}\left\{p_{j}\right\}$ dos tempos de processamento das tarefas sendo $j=1,2, \ldots, n$;

Passo 2 - Criar $k$ intervalos, com tamanhos aproximadamente iguais, variando entre $[p, \bar{p}]$, o valor de $k$ é definido segundo a tabela 1. Para cada processador, associar um conjunto $I$ de intervalos, representados por $I_{1}, I_{2}, \ldots, I_{k}$;

Passo 3 - Enquanto existirem tarefas a serem alocadas, faça: Determine o intervalo $I_{l}$ que contém a tarefa $j$. Aloque $j$ no intervalo $I_{l}$ do processador que apresentar a menor relação entre o número de tarefas alocadas no intervalo $\left(n\left(I_{l}\right)\right)$, pela velocidade deste processador $\left(\sigma_{i}\right)$. Em caso de igualdade na relação, a tarefa é alocada ao processador de maior velocidade.

Fase 2

Passo1 - Identifique os processadores mais e menos carregados e chame-os de $M_{1}$ e $M_{m}$, respectivamente.

Passo 2 - Considerando: $C_{1}$ a carga do processador mais carregado; $C_{m}$ a carga do processador menos carregado; e $\bar{C}=\sum_{j=1}^{n} p_{j} / \sum_{i=1}^{m} \sigma_{i}$ o tempo médio de finalização, utilizado como limitante inferior nos resultados apresentados ao final[5]. Calcule $\Delta_{1}=C_{1}-\bar{C}, \Delta_{m}=\bar{C}-C_{m}, \Delta=\min \left\{\Delta_{1}-\Delta_{m}\right\}$ e $\Delta^{\prime}=C_{1}-C_{m}$.

Se $\sigma_{m} \Delta<p$, não existem tarefas que produzem um makespan melhor, portanto a Fase 2 termina.

Se $\sigma_{m} \Delta>\bar{p}$ faça $l=k$ (o maior intervalo).

Caso contrário, $\sigma_{m} \Delta$ pertence a algum intervalo $I_{l}$.

Passo 3 - Se nenhuma tarefa $j$ com $p_{j} \in I_{l}$ e $p_{j} \leq \sigma_{m} \Delta$ está alocada em $M_{1}$, vá para o Passo 4. Caso contrário mova a primeira tarefa encontrada, dita $l$, de $M_{1}$ para o intervalo $I_{l}$ em $M_{m}$ e volte para o Passo 1.

Passo 4 - Se nenhuma tarefa $j$ com $p_{j} \in I_{l^{-}}=I_{l} \cup I_{2} \cup \ldots \cup I_{l-1}$ está alocada em $M_{1}$, vá para o passo 5. Caso contrário, mova a primeira tarefa encontrada com $p_{j} \in I_{l^{-}}$de $M_{1}$ para o intervalo $I_{l^{-}}$em $M_{m}$ e volte para o Passo 1.

Passo 5 - Se $l=k$ vá para Fase 3 .

Se nenhuma tarefa $j \operatorname{com} p_{j} \in I_{l^{+}}=\left\{\cup I_{i}, i=l, l+1, \ldots \mid p_{i}<\sigma_{m} \Delta^{\prime}\right.$ ou $\left.i=k\right\}$ e $p_{j}<\sigma_{m} \Delta^{\prime}$, vá para a Fase 3 , caso contrário, mova uma tarefa de $I_{l^{+}}$de $M_{1}$ para $M_{m}$ e vá para o Passo 1 .

Fase 3

Passo 1 - Para cada processador $h=m, m-1, \ldots$, calcular $\theta=\frac{\sigma_{1} \cdot \sigma_{h}\left(C_{1}-C_{h}\right)}{\sigma_{1}+\sigma_{h}} \mathrm{e}$ identifique o par de tarefas $j$ e $j^{\prime}$ onde $\left|p_{j}-p_{j}{ }^{\prime}\right|$ é mais próximo ao valor de $\theta$ de tal forma que $p_{j}{ }^{\prime}<p_{j}$ e que $p_{j}{ }^{\prime}>p_{j}-\left(C_{1}-C_{h}\right) / \sigma_{h}$. Caso um par de tarefas que satisfaça a condição for encontrado, realize a troca e volte à Fase 1. Caso contrário o algoritmo termina. 


\section{A heurística IProc}

Visando melhorar a qualidade de solução sem comprometer o tempo computacional, foram estudados melhoramentos para a versão original da heurística Kproc, principalmente no tocante aos conceitos de maior relevância presentes nesse procedimento: a distribuição das tarefas em intervalos e a estratégia utilizada para a busca local.

Após uma avaliação dos valores de $k$ que apresentam melhor relação entre desempenho e qualidade da solução, foi proposta uma função contínua baseada nos valores empíricos discretos definidos por [7]. Como indicado abaixo:

$$
k=30 \cdot \log (1+5(x / 1000))+5,
$$

onde $x=n / m$, ou seja, é a relação entre o número de tarefas e o de processadores.

Num segundo momento, realizou-se uma modificação na fase de balanceamento da carga (Fase 2), alterando a estratégia de busca por tarefas candidatas à troca, que originalmente selecionava a primeira tarefa cuja troca reduzisse a carga do processador mais carregado, por uma política onde faz-se uma simulação do efeito da troca aos processadores envolvidos e escolhe-se a que minimizar a carga simulada do processador mais carregado.

Na Fase 3, modificou-se o valor alvo de busca das tarefas candidatas à troca dupla do método original por um procedimento que, como o anterior, calcula o efeito da permutação das tarefas selecionadas aos processadores envolvidos e seleciona o par de tarefas que minimizar a carga estimada do processador mais carregado.

\section{Fases do Iproc}

Fase 1

A única alteração nessa fase refere-se à função que determina o número de intervalos, que passam a ser calculados através da equação (3.1).

\section{Fase 2}

Passo 1 - Identifique os processadores mais e menos carregados $\left(M_{1}\right.$ e $M_{m}$, respectivamente).

Passo 2 - Calcule $\Delta=C_{1}-C_{m}$ e $\Phi=\sigma_{m} \Delta$, onde $\Phi$ será tratado como um valor limitante para a busca

Passo 3 - Se $\Phi<\underline{p}$, a Fase 2 termina. Caso contrário, identifique o intervalo que contém o valor de $\Phi$ e chame de $I_{f}$. Procure por tarefas alocadas em $M_{1}$ com $p_{j} \in I_{f}(f=f, f-1, \ldots, 1)$ e $p_{j} \leq \Phi$. Se tais tarefas não existirem, a Fase 2 termina. Senão, selecione a tarefa que minimizar o valor da função dife, representada a seguir.

$$
\operatorname{dife}=\left|\bar{C}-\left(C_{m}-p_{j} / \sigma_{m}\right)\right|,
$$

onde: $p_{j}=$ tempo de processamento de uma tarefa do processador mais carregado;

$\sigma_{m}=$ velocidade do processador menos carregado;

$\bar{C}=$ tempo médio de finalização, proposto por [5].

Realoque a tarefa $j$, selecionada, de $M_{1}$ para o intervalo $I_{f}$ em $M_{m}$ e volte para o Passo 1. 
Fase 3

Passo 1 - Identifique os processadores mais e menos carregados $\left(M_{1}\right.$ e $M_{m}$, respectivamente) e nomeie arbitrariamente os processadores restantes como sendo: $m-1, m-2, \ldots, 2$ onde $m$ é o número de processadores.

Passo 2 - Se $m=1$, finaliza a Fase 3. Caso contrário, para cada tarefa $j$, do processador $M_{1}$, procure, em $M_{m}$, uma tarefa $j^{\prime}$ que satisfaça as seguintes condições: $p_{j}{ }^{\prime}<p_{j}$ e $p_{j}{ }^{\prime}>p_{j}-\left(C_{1}-C_{m}\right) / \sigma_{m}$. Caso não existir nenhum par de tarefas que satisfaça as condições, faça $m=m-1$ e retorne ao Passo 2.

Caso contrário, para cada par de tarefas encontrado, calcule: $C_{1}{ }^{\prime}=C_{1}-\left(p_{j}-\right.$ $\left.p_{j}{ }^{\prime}\right) / \sigma_{1}, C_{m}{ }^{\prime}=C_{m}-\left(p_{j}-p_{j}{ }^{\prime}\right) / \sigma_{m}$ e $\operatorname{swap}=\left\{\max \left(C_{1}{ }^{\prime}, C_{m}{ }^{\prime}\right)\right\}$

Selecione o par que minimizar o valor da função swap, realize a permutação das tarefas selecionadas e volte para a Fase 2.

Antes de se apresentar os resultados computacionais, vai-se apresentar resumidamente o ambiente CORE, onde todos os algoritmos e instâncias aqui apresentados estão disponíveis, tanto para execução remota quanto para download.

\section{O projeto CORE}

O projeto CORE (Combinatorial Optimization Resource Management Environment) consiste em um ambiente acessível pela Internet que suporta a execução remota distribuída de diferentes métodos de resolução de problemas de otimização combinatória.

O ambiente oferece um conjunto de métodos heurísticos e metaheurísticos, que podem ser dinamicamente combinados para a obtenção de melhores resultados na solução de problemas de grande interesse científico e industrial.

Utilizando-se interfaces gráficas de fácil utilização, é possível a criação de planos de otimização, que consistem em estratégias para a solução de problemas (seleção de métodos e configuração de seus respectivos parâmetros) que serão executados sobre instâncias de teste ou instâncias enviadas pelo usuário. O ambiente foi desenvolvido através do paradigma de orientação a objetos e objetos distribuídos[2].

Mais informações sobre o projeto CORE podem ser encontradas em [10] e na página do projeto (http://glover.ce.ufsm.br).

\section{Resultados Computacionais}

Para avaliação do método Iproc proposto, utilizou-se a comparação com o método Kproc original. Os dois métodos foram comparados com o limitante inferior proposto por [5]. Foram geradas 10 instâncias para cada combinação de $m=2$ e 3 e $n=10,50,100,500$ e 1000; e $m=5,7,10,15$ e 20 e $n=50,100,500$ e 1000, com tempos de processamento sendo gerados de forma uniformemente distribuída nos intervalos $[1,100],[1,1000]$ e $[1,1000]$, considerando um conjunto com números inteiros e outro com números reais, totalizando 1800 instâncias. As velocidades das máquinas foram geradas obedecendo a uma distribuição uniforme no intervalo $[2,20]$. 
Como os resultados obtidos para as diversas amplitudes de tempos de processamento apresentaram resultados semelhantes, vão-se apresentar apenas os referentes aos dados com tempos de processamento no intervalo [1,100]. A tabela 2 mostra os resultados para cada combinação de $m$ e $n$, onde $s_{1}$ é a média da diferença percentual entre o valor de função objetivo obtido pelo método Kproc e o limitante inferior, para as 10 instâncias consideradas; $s_{2}$ é a média da diferença percentual entre o valor de função objetivo obtido pelo método Iproc e o limitante inferior, para as 10 instâncias consideradas; $t_{1}$ é a média do tempo computacional (em segundos) para execução de Kproc, para as 10 instâncias consideradas e $t_{2}$ é a média do tempo computacional (em segundos) para execução de Iproc, para as 10 instâncias consideradas.

Os testes foram executados em um computador Pentium III 1 Gigahertz, com 384 Megabytes de $R A M$, sob o sistema operacional Linux com kernel 2.4.3.

Observou-se que, na quase totalidade dos casos, o desempenho de Iproc foi superior a Kproc as custas de um mínimo acréscimo de esforço computacional. Os casos onde isso não ocorreu se referem a $(m, n)=(2,50)$ e $(3,100)$ com tempos de processamento reais uniformemente distribuídos nos intervalos $[1,1000]$ e $[1,10000]$, respectivamente.

São apresentados também, em forma de gráficos, os desempenhos médio, máximo e o desvio padrão de $\operatorname{Kproc}\left(s_{1}\right)$ e $\operatorname{Iproc}\left(s_{2}\right)$ para $m=2,3,5,7,10,15$ e 20 e $n$ $=50$ e 100 (Figuras 1 e 2, respectivamente) com tempos de processamento inteiros uniformemente distribuídos no intervalo [1,100]. Pode-se observar que Iproc apresenta sempre um melhor desempenho médio, menor desvio padrão e menor resultado máximo, quando comparado ao Kproc, demonstrando sua robustez. Para se demonstrar as mesmas conclusões é apresentada, ainda, a Figura 3, que demonstra o desempenho médio de $\operatorname{Kproc}\left(s_{1}\right)$ e $\operatorname{Iproc}\left(s_{2}\right)$ para valores de tempos de processamento inteiros.

\section{Conclusões}

A qualidade de solução da heurística Iproc foi superior em todas as combinações, excetuando-se a relação processador/tarefa $(2,50)$ das tarefas geradas no intervalo $[1,100]$ e as relações $(3,100)$ e $(3,10)$ do intervalo de tarefas $[1,10000]$, o que representa um ganho em qualidade de solução de 99,98\% dos casos. O tempo computacional, no entanto, foi em média $20 \%$ superior ao da heurística Kproc, o que deve-se a exploração mais refinada das trocas possíveis na Fase 2. Portanto, pode-se dizer que o método apresentou melhores resultados, tanto médios quanto máximos às custas de um pequeno esforço computacional adicional.

A disponibilização dos métodos através do ambiente CORE facilitou a execução dos testes e acelerou o processo de desenvolvimento, permitindo que um grupo maior de usuários participasse do processo. Além disso, obteve-se reutilização de código e 


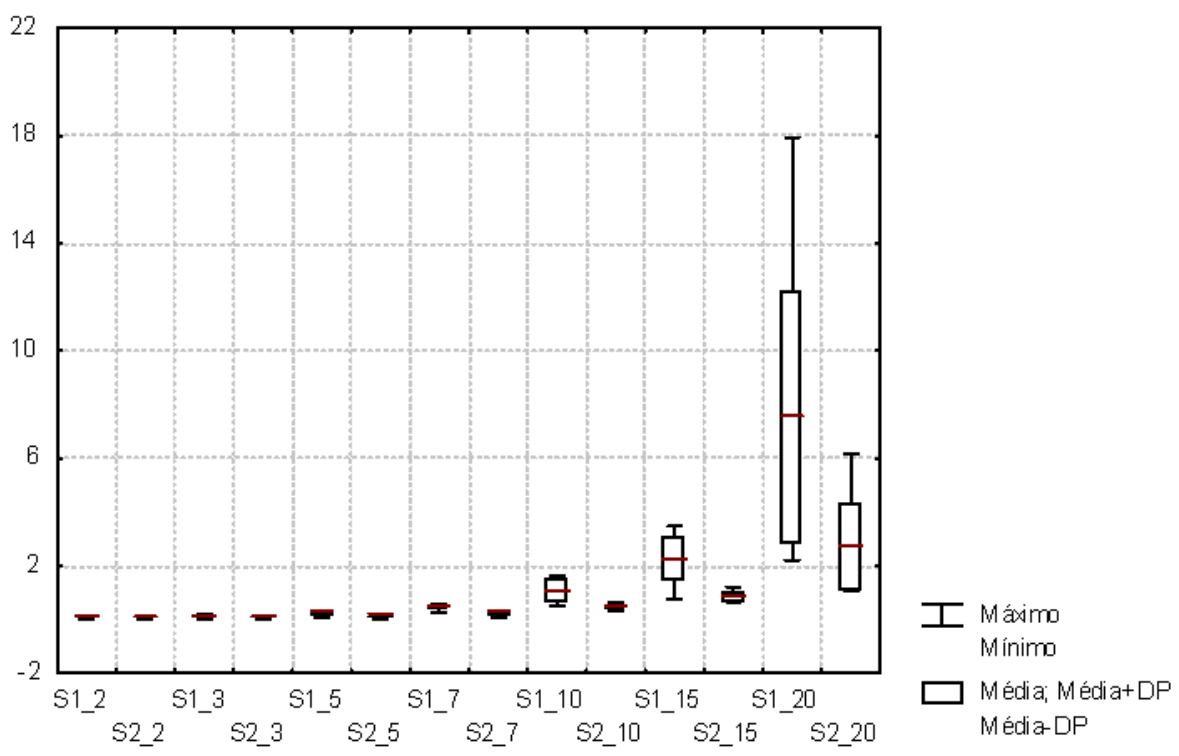

Figura 1: Desempenho de $\operatorname{Kproc}\left(s_{1}\right)$ e $\operatorname{Iproc}\left(s_{2}\right)$ para $\mathrm{n}=50 \mathrm{com}$ tempos de processamentos inteiros uniformemente distribuídos no intervalo $[1,100]$

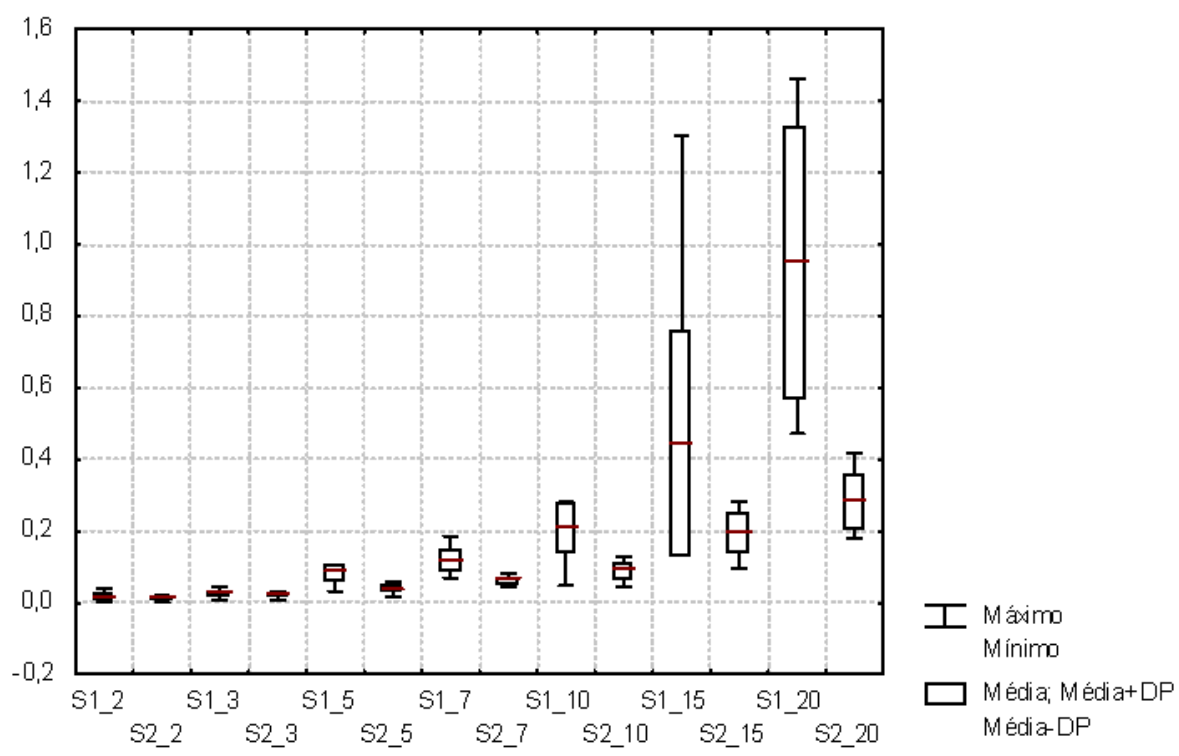

Figura 2: Desempenho de $\operatorname{Kproc}\left(s_{1}\right)$ e $\operatorname{Iproc}\left(s_{2}\right)$ para $\mathrm{n}=100 \mathrm{com}$ tempos de processamentos inteiros uniformemente distribuídos no intervalo $[1,100]$ 
Tabela 2: Resultados considerando tempos de processamento gerados entre $[1,100]$

\begin{tabular}{|c|c|c|c|c|c|c|c|c|c|}
\hline$m$ & $n$ & \multicolumn{4}{|c|}{ Instâncias Inteiras } & \multicolumn{4}{|c|}{ Instâncias Reais } \\
\hline & & $s_{1}$ & $s_{2}$ & $t_{1}$ & $t_{2}$ & $s_{1}$ & $s_{2}$ & $t_{1}$ & $t_{2}$ \\
\hline \multirow[t]{5}{*}{2} & 10 & 0,49386 & 0,22125 & 0,4 & 0,5 & 1,92782 & 0,30276 & 0,3 & 0,6 \\
\hline & 50 & 0,04359 & 0,02791 & 1,4 & 1,3 & 0,00850 & 0,00379 & 2,1 & 2,4 \\
\hline & 100 & 0,01320 & 0,00962 & 2,6 & 6,5 & 0,00313 & 0,00032 & 3,4 & 4,0 \\
\hline & 500 & 0,00253 & 0,00141 & 13,3 & 10,3 & 0,00005 & 0,00004 & 16,0 & 20,7 \\
\hline & 1000 & 0,00265 & 0,00110 & 15,2 & 22,8 & 0,00002 & 0,00001 & 37,7 & 44,1 \\
\hline \multirow[t]{5}{*}{3} & 10 & 3,15542 & 1,65902 & 0,5 & 0,5 & 4,79070 & 1,71343 & 0,7 & 0,4 \\
\hline & 50 & 0,06562 & 0,03705 & 2,3 & 1,8 & 0,04508 & 0,01188 & 2,9 & 3,7 \\
\hline & 100 & 0,02343 & 0,01679 & 3,4 & 2,7 & 0,00539 & 0,00229 & 5,0 & 6,6 \\
\hline & 500 & 0,00723 & 0,00441 & 14,6 & 14,3 & 0,00006 & 0,00006 & 34,9 & 32,3 \\
\hline & 1000 & 0,00487 & 0,00151 & 29,6 & 31,4 & 0,00002 & 0,00001 & 80,0 & 71,4 \\
\hline \multirow[t]{4}{*}{5} & 50 & 0,18572 & 0,09536 & 2,9 & 3,4 & 0,16600 & 0,07821 & 4,1 & 4,3 \\
\hline & 100 & 0,08097 & 0,03556 & 4,9 & 4,7 & 0,01329 & 0,00656 & 6,7 & 8,2 \\
\hline & 500 & 0,01429 & 0,00840 & 15,8 & 17,3 & 0,00026 & 0,00006 & 42,2 & 42,2 \\
\hline & 1000 & 0,00747 & 0,00433 & 33,0 & 36,1 & 0,00006 & 0,00004 & 81,3 & 81,6 \\
\hline \multirow[t]{4}{*}{7} & 50 & 0,39191 & 0,19554 & 3,4 & 3,4 & 0,28623 & 0,07820 & 5,3 & 5,6 \\
\hline & 100 & 0,11572 & 0,05999 & 5,3 & 5,9 & 0,04635 & 0,01339 & 9,0 & 12,5 \\
\hline & 500 & 0,02379 & 0,01197 & 15,1 & 22,1 & 0,00052 & 0,00016 & 54,0 & 51,3 \\
\hline & 1000 & 0,01312 & 0,00540 & 35,5 & 42,1 & 0,00010 & 0,00006 & 86,6 & 95,5 \\
\hline \multirow[t]{4}{*}{10} & 50 & 1,04593 & 0,40876 & 6,3 & 3,9 & 0,84235 & 0,30853 & 5,3 & 6,2 \\
\hline & 100 & 0,20711 & 0,08898 & 6,7 & 7,6 & 0,12483 & 0,04851 & 9,9 & 15,4 \\
\hline & 500 & 0,03408 & 0,02058 & 20,1 & 24,9 & 0,00109 & 0,00034 & 48,6 & 71,9 \\
\hline & 1000 & 0,01941 & 0,00886 & 35,6 & 64,3 & 0,00023 & 0,00017 & 96,0 & $\overline{100,6}$ \\
\hline \multirow[t]{4}{*}{15} & 50 & 2,21228 & 0,82429 & 8,9 & 7,2 & 2,04068 & 0,99937 & 8,1 & 9,0 \\
\hline & 100 & 0,44051 & 0,19147 & 9,4 & 10,2 & 0,28191 & 0,10111 & 17,3 & 22,0 \\
\hline & 500 & 0,04949 & 0,02732 & 23,6 & 30,5 & 0,00276 & 0,00097 & 90,1 & 86,9 \\
\hline & 1000 & 0,02741 & 0,01355 & 42,5 & 73,3 & 0,00048 & 0,00018 & 124,7 & 153,4 \\
\hline \multirow[t]{4}{*}{20} & 50 & 7,46746 & 2,64307 & 8,9 & 11,1 & 6,02549 & 2,29970 & 10,3 & 14,0 \\
\hline & 100 & 0,94493 & 0,27985 & 13,0 & 14,5 & 0,72756 & 0,20102 & 17,4 & 27,2 \\
\hline & 500 & 0,07607 & 0,04012 & 26,4 & 35,9 & 0,00680 & 0,00174 & 113,1 & 145,1 \\
\hline & 1000 & 0,03791 & 0,01869 & 52,9 & 78,5 & 0,00076 & 0,00018 & 195,4 & 169,0 \\
\hline
\end{tabular}


projeto no desenvolvimento dos métodos heurísticos.

\title{
Agradecimentos
}

Os autores agradecem as sugestões feitas pelo assessor que revisou o artigo, pois foram de grande valia para uma melhor compreensão do texto. O trabalho de Felipe Martins Müller foi parcialmente financiado pelo CNPq.

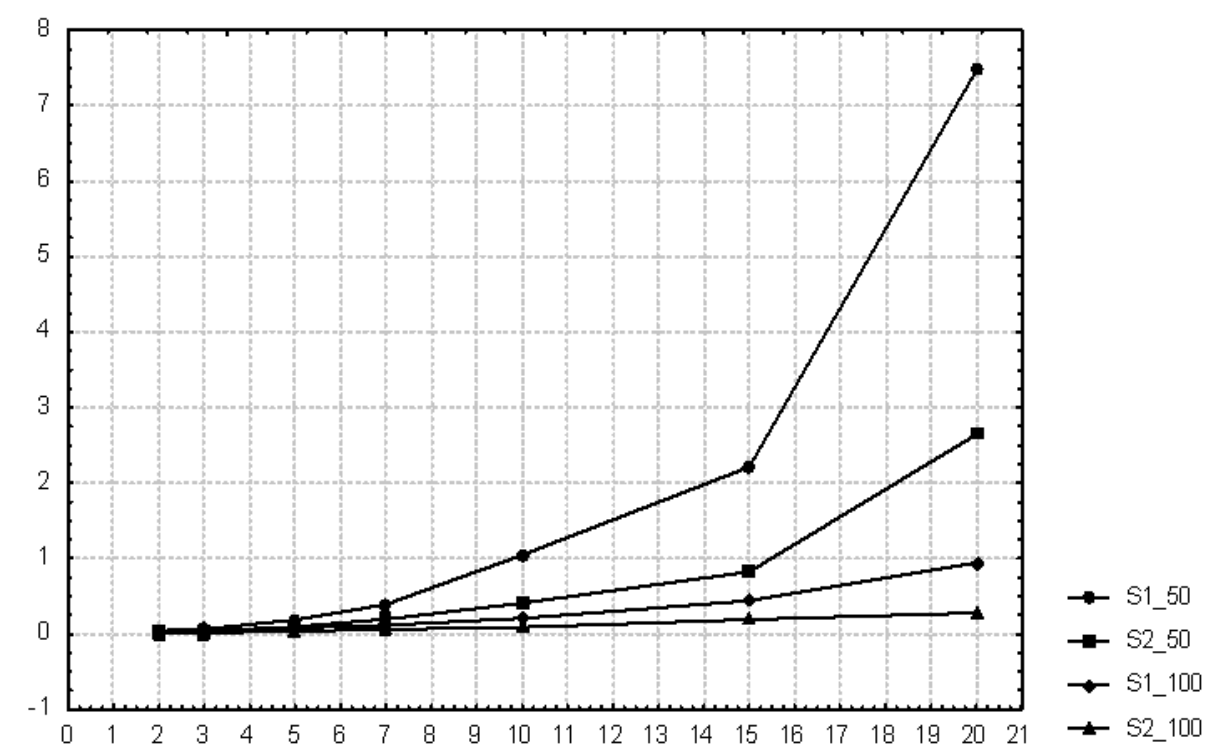

Figura 3: Gráfico comparativo do desempenho médio de $\operatorname{Kproc}\left(s_{1}\right)$ e $\operatorname{Iproc}\left(s_{2}\right)$ para $\mathrm{n}=50$ e $\mathrm{n}=100$, tempos de processamento inteiros uniformemente distribuídos no intervalo $[1,100]$

\begin{abstract}
.
This paper deals with the problem of assigning a limited set of tasks to a given set of machines with different processing speeds, with no preemption and aiming to minimize the total execution time (makespan). The solution approach chosen in this paper was the Kproc heuristic, in which improvements were proposed, resulting in a solution quality gain in 99,98\% of the instances types considered in the experiments, without a significant computation time increase. Still, Kproc, Kprocand many other heurístic and metaheuristc methods were implemented using the CORE (Combinatorial Optimization Resource Management Environment) framework, which allows the distributed remote execution of combinatorial optimization resolution methods through the Internet structure. The CORE environment can be accessed from: http://glover.ce.ufsm.br.
\end{abstract}




\section{Referências}

[1] G. Dobson, Scheduling independent tasks on uniform processors. SIAM Journal on Computing, 13 (1984), 705-716.

[2] J. Farley, "Java Distributed Computing", O'Reilly, USA, 1998.

[3] T. Gonzalez, O.H. Ibarra e S. Sahni, Bounds for LPT schedules on uniform processors, SIAM Journal on Computing, 6 (1977), 155-166.

[4] R.L. Graham, E.L. Lawler e J.K. Lenstra, Optimization and aproximation in determinist sequencing: a survey, Annals of Discrete Mathematics, 5 (1979), 287-326.

[5] M. Kunde, A MULTIFIT algorithm for uniform multiprocessor scheduling, Lecture Notes in Computer Science, 145 (1983), 175-185.

[6] E.L. Lawler, J.K. Lenstra, A.H.G. Rinnooy Kan e D.B. Shmoys, Sequencing and scheduling: algorithms and complexity, Report BS-R8909, Center of Mathematics and Computer Science, 1989, Amsterdan.

[7] S. J. Limberger e F. M. Müller, Uma heurística de trocas para o problema de seqüenciamento de tarefas em processadores uniformes, Pesquisa Operacional, 20 (2000), 31-42.

[8] J.F. Morrison, A note on LPT scheduling, Operations Research Letters, 7 (1988), 77-79.

[9] F.M. Müller, "Algoritmos Heurísticos e Exatos para Resolução do Problema de Seqüenciamento em Processadores Paralelos", Tese de Doutorado, DENSIS, UNICAMP, Campinas, SP, 1993.

[10] F.M. Müller e H.G. Santos, CORE - an internet based combinatorial optimization service provider em "Technical and Organizational Integration of Supply Chains (F. S. Flogliato et al., Eds.)", Proceedings os the XXII ICIEOM, (2002), 113-120. 